\title{
Hysteroscopic intrauterine morcellation of submucosal fibroids: preliminary results in Hong Kong and comparisons with conventional hysteroscopic monopolar loop resection
}

\author{
Menelik MH Lee *, Tomoko Matsuzono
}

\section{A B S T R A C T}

Introduction: Hysteroscopic management of submucosal fibroids using the intrauterine morcellation technique is increasingly being adopted worldwide but no literature concerning its safety and efficacy is available within our local population. We aimed to determine the safety, satisfaction, and efficiency of hysteroscopic intrauterine morcellation of submucosal fibroids, and to compare this technique with conventional hysteroscopic monopolar loop resection to identify its potential benefits.

Methods: All cases of hysteroscopic resection of submucosal fibroids performed in a regional hospital in Hong Kong between 1 January 2011 and 31 December 2014, either by hysteroscopic intrauterine morcellation (MyoSure; Hologic, Bedford [MA], US) or conventional hysteroscopic monopolar loop

- A video clip showing hysteroscopic intrauterine morcellation of submucosal fibroids is available at $<$ sww.hkmj.org >

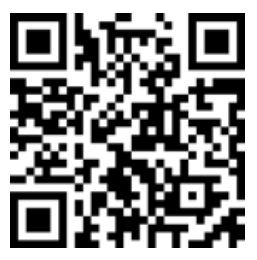

This article was published on 8 Jan 2016 at www.hkmj.org. resection, were selected and case notes reviewed. Technical details such as fibroid size, operating time, fluid deficit, operative complications, patient satisfaction, and improvement in haemoglobin level were analysed and compared between the hysteroscopic intrauterine morcellation and the conventional groups. All statistical results were calculated using the Mann-Whitney test.

Results: During the 3-year period, 29 cases of submucosal fibroids were managed by hysteroscopic surgery. Conventional hysteroscopic monopolar loop resection was performed in 14 patients and another 15 underwent hysteroscopic intrauterine morcellation with the MyoSure device. At 3-month follow-up, there was no significant difference in overall patient satisfaction $(84.6 \%$ for conventional method vs $93.3 \%$ for hysteroscopic intrauterine morcellation method; $\mathrm{P}=0.841$ ). Both techniques showed improvement in haemoglobin level at 3 months but without significant difference between the two groups: $+21.5 \mathrm{~g} / \mathrm{L}(+1$ to $+44 \mathrm{~g} / \mathrm{L})$ for conventional group and $+17.0 \mathrm{~g} / \mathrm{L}(-4$ to +40 $\mathrm{g} / \mathrm{L})$ for hysteroscopic intrauterine morcellation group $(\mathrm{P}=0.235)$. Both techniques achieved $100 \%$ satisfaction if the submucosal fibroid had over $60 \%$ of its contents protruding into the uterine cavity. The operating time was significantly reduced for the hysteroscopic intrauterine morcellation technique (mean, 36.6 mins vs 53.6 mins in conventional hysteroscopic monopolar loop resection; $\mathrm{P}=0.005$ ), particularly in those whose fibroids were $\leq 3.0 \mathrm{~cm}$ (mean, 27.6 mins vs 53.4 mins; $\mathrm{P}=0.019$ ).

Conclusions: This retrospective review suggests that hysteroscopic intrauterine morcellation of submucosal fibroids is a safe and effective method in the management of menorrhagia in Chinese women. Preliminary data suggest this technique to be less time-consuming, especially when managing fibroids of $\leq 3.0 \mathrm{~cm}$.

\section{Hong Kong Med J 2016;22:56-61 \\ DOI: $10.12809 / \mathrm{hkmj} 154600$}

MMH Lee *, FHKCOG, FHKAM (Obstetrics and Gynaecology)

T Matsuzono, FHKCOG, FHKAM (Obstetrics and Gynaecology)

Department of Obstetrics and Gynaecology, Queen Elizabeth Hospital, Jordan, Hong Kong

* Corresponding author: menelik.lee@gmail.com

New knowledge added by this study

- Hysteroscopic intrauterine morcellation of submucosal fibroids is as effective as conventional hysteroscopic resection of submucosal fibroids. The operating time is shorter than the conventional technique, particularly when the submucosal fibroid is $\leq 3.0 \mathrm{~cm}$.

Implications for clinical practice or policy

- Reduced operating time for management of submucosal fibroids will enable more such procedures to be performed within a set limit of time. This may reduce waiting time for surgery and improve overall patient satisfaction, particularly in a public hospital setting.

\section{Introduction}

Submucosal fibroids are a common cause of heavy menstrual bleeding. ${ }^{1}$ Traditionally, conventional hysteroscopic monopolar loop resection of such fibroids represents the surgical treatment of choice (Fig 1a). Hysteroscopic management of such fibroids 
using the intrauterine morcellation (IUM) technique (eg MyoSure, Hologic, Bedford, US; Truclear, Smith \& Nephew Inc, Andover, US; Fig 1b), however, is increasingly being used worldwide but no literature concerning its safety and efficacy is available within our local population.

Our hospital is one of the first to introduce the use of the hysteroscopic IUM device in Hong Kong. This preliminary review looks at the safety, satisfaction, and efficiency of such technique when performed within our hospital and within a Chinese population. Comparisons were made between the conventional hysteroscopic monopolar loop resection technique and IUM technique with the aim of identifying the potential benefits that have been described by previous studies worldwide.

\section{Methods}

All cases of hysteroscopic resection of submucosal fibroids performed at Queen Elizabeth Hospital, Hong Kong, between 1 January 2011 and 31 December 2014, either by IUM (MyoSure) or conventional hysteroscopic monopolar loop resection, were selected and the case notes were reviewed. Choice of method was dependant on the operator but all cases using the IUM method were performed between the years 2013 and 2014 after its introduction in our department. Patients were identified from the Clinical Data Analysis and Reporting System through specialised coding. Detailed technical aspects of both operations were collected using a self-designed proforma with information collected via the Clinical Management System computerised record system. Analysis and comparison of technical details such as fibroid size, operating time, fluid deficit, operative complications, and patient satisfaction were made between the IUM and the conventional groups. Fibroid size was measured via preoperative abdominal and/or vaginal fluid-infused sonography, and confirmed during diagnostic hysteroscopy prior to resection. Those

\section{在香港使用宮腔鏡粉碎術切除粘膜下肌瘤的初步 成果, 並與傳統宮腔鏡單極環切除術進行比較}

\section{李文軒、松園知子}

引言：以宮腔鏡粉碎術切除粘膜下肌瘤在各地越趨普遍, 但有關其應 用於本地人口的安全性和效用的文獻仍未有記載。本研究旨在探討宮 腔鏡粉碎術切除粘膜下肌瘤的安全性、滿意度和效率, 並與傳統宮腔 鏡單極環切除術比較, 以確定它的潛在好處。

方法：回顧2011年1月1日至2014年12月31日期間於香港一所分區醫 院進行宮腔鏡切除粘膜下肌瘤的所有患者的病歷紀錄。患者均接受 宮腔鏡粉碎術（MyoSure）或傳統宮腔鏡單極環切除術。分析並比較 兩種技術的細節, 如子宮肌瘤大小、手術時間、體液流失、手術併 發症、病人滿意度和血紅蛋白水平的改善。所有統計結果均以MannWhitney檢驗來進行計算。

結果：3年內共有 29 例經宮腔鏡手術治理粘膜下肌瘤, 其中 14 例進 行傳統切除術, 另 15 例利用MyoSure裝置進行宮腔鏡粉碎術。經過 3 個月隨訪, 兩種技術的整體病人滿意度無顯著差異：傳統方法為 $84.6 \%$, 宮腔鏡粉碎術為 $93.3 \%(\mathrm{P}=0.841)$ 。兩組的血紅蛋白 水平均有改善, 但未達顯著差異：傳統方法上升了 $21.5 \mathrm{~g} / \mathrm{L}(+1$ 至 $+44 \mathrm{~g} / \mathrm{L})$ ，宮腔鏡粉碎術上升了 $17.0 \mathrm{~g} / \mathrm{L}(-4$ 至 $+40 \mathrm{~g} / \mathrm{L})$ ； $\mathrm{P}=0.235$ 。如果黏膜下肌瘤有 $60 \%$ 突入宮腔, 兩種技術的病人滿意 度均達至 $100 \%$ 。宮腔鏡粉碎術的手術時間明顯比傳統切除術短, 前者平均為36.6分鐘, 後者為53.6分鐘 $(\mathrm{P}=0.005)$, 尤其是對於 $3.0 \mathrm{~cm}$ 或以下的肌瘤：前者平均手術時間為 27.6 分鐘, 後者為53.4分 鐘 $(\mathrm{P}=0.019)$

結論：本回顧研究顯示對於月經過多的華籍女性來説, 針對粘膜下肌 瘤的宮腔鏡粉碎術是一項既安全又有效的方法。初步數據顯示尤其是 對於 $3.0 \mathrm{~cm}$ 或以下的肌瘤, 這項新技術耗時較少。

cases with prolonged operating time due to multiple operations for other indications or complications were excluded from final analysis. In our hospital, monopolar energy was used during conventional loop resection, hence glycine was used as the distending medium. With the IUM technique, since no energy source was required during morcellation, normal saline was used as the distending medium. Operating
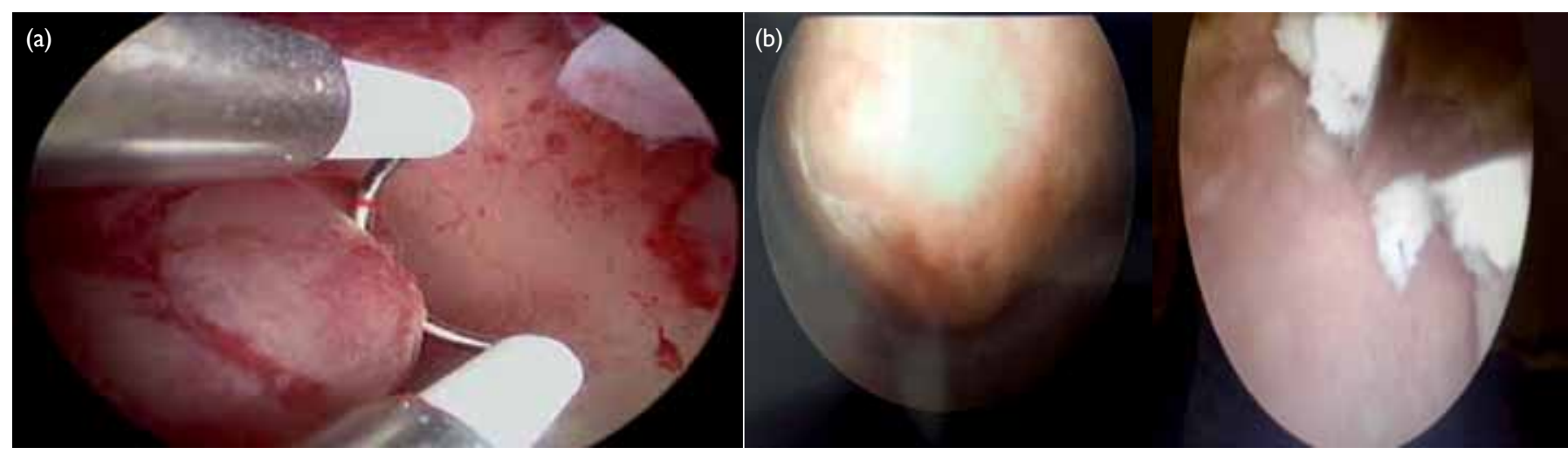

FIG I. (a) Conventional hysteroscopic loop resection of submucosal fibroid, and (b) intrauterine morcellation of submucosal fibroid 
time was measured from the time the patient was anaesthetised to completion of the operation. Hence operating time included the time required to position the patient, cleaning, draping, and the time for setting up equipment. In the IUM system, fluid deficit calculations were accurately measured by the Aquilex Fluid Control System (Hologic, Bedford, US). With the conventional method, deficit calculations were based on the amount of fluid entered minus the amount of fluid suctioned and retrieved intraoperatively. Postoperatively, a satisfactory outcome was considered when the patient subjectively reported reduced menstrual bleeding and considered the operation to have improved menstrual symptoms at 3 months' follow-up. Pre- and postoperative haemoglobin levels within 3 months of follow-up and differences between them were also investigated. All results were statistically analysed using the Statistical Package for the Social Sciences (Windows version 22.0; SPSS Inc, Chicago [IL], US). Statistical significance was represented by $\mathrm{P}$ values that were calculated using the Chi squared test for patient satisfaction and Mann-Whitney test for the remaining tests. $\mathrm{P}$ values of $<0.05$ were considered statistically significant for all of the data.

The research protocol was approved by the Ethics Committee of the study hospital. The patients were not required to undergo additional tests or visits, and therefore consent from the patients was not required.

\section{Results}

During the study period, 29 patients with submucosal fibroids were treated with hysteroscopic surgery at Queen Elizabeth Hospital, Hong Kong. Conventional hysteroscopic monopolar loop resection was performed in 14 patients and IUM with the MyoSure device in 15.

Among those who underwent surgery using the conventional technique, one patient experienced a uterine perforation that required surgical repair and was excluded from data analysis. No complications occurred in any patient in the IUM group. Two patients from the IUM group and one from the conventional group were excluded due to the need for multiple procedures including endometrial ablation or laparoscopic ovarian cystectomy during the same operation. The remaining patients were all well and discharged the day after their operation regardless of the hysteroscopic technique used.

The mean size of fibroids resected was $3.3 \mathrm{~cm}$ (range, 2-5 cm; median, $3 \mathrm{~cm}$ ) for the conventional technique and 3.5 (range, 1.5-6 cm; median, 3 $\mathrm{cm})$ for the IUM group, although they were not significantly different $(\mathrm{P}=0.470)$. The operating time was significantly shorter using the IUM technique (mean, 36.6 mins; range, 17-72 mins) compared with the conventional technique (mean, 53.6 mins; range, 39-102 mins) $[\mathrm{P}=0.005]$. Total fluid deficit, however, was significantly greater when using the IUM technique (1005 $\mathrm{mL}$; range, 40-2600 $\mathrm{mL}$ ) compared with the conventional technique $(225 \mathrm{~mL}$; range, $0-1000 \mathrm{~mL})[\mathrm{P}=0.003$; Table 1$]$. No patient in either group developed any complication associated with excessive fluid absorption.

At 3 months' follow-up, there was no significant difference in the overall outcome between the two groups: $84.6 \%$ of patients who underwent the conventional method versus $93.3 \%$ of those who underwent IUM were pleased with their overall outcome $(\mathrm{P}=0.841)$. Within the conventional group, the mean preoperative haemoglobin level was 95.4 $\mathrm{g} / \mathrm{L}$ (range, 81-121 g/L). The mean postoperative haemoglobin level of nine patients who returned

TABLE I. Comparison of conventional hysteroscopic monopolar loop resection technique with the hysteroscopic intrauterine morcellation technique

\begin{tabular}{|c|c|c|c|}
\hline & $\begin{array}{l}\text { Conventional hysteroscopic } \\
\text { monopolar loop resection }(n=14)\end{array}$ & $\begin{array}{l}\text { Hysteroscopic morcellation } \\
\text { (MyoSure) [n=15] }\end{array}$ & $P$ value \\
\hline Complications & 1 (Uterine perforation) & None & - \\
\hline Excluded cases & $\begin{array}{l}2 \text { (1 Uterine perforation and } \\
1 \text { additional contaminant procedure) }\end{array}$ & $\begin{array}{l}2 \text { (Additional contaminant } \\
\text { procedure) }\end{array}$ & - \\
\hline Total No. of patients in data analysis & 12 & 13 & \\
\hline Mean (range) fibroid size $(\mathrm{cm})$ & $3.3(2-5)$ & $3.5(1.5-6)$ & 0.470 \\
\hline Duration (range) of procedure (minutes) & $53.6(39-102)$ & $36.6(17-72)$ & 0.005 \\
\hline Mean (range) fluid deficit (mL) & $225(0-1000)^{\star}$ & 1005 (40-2600)† & 0.003 \\
\hline Satisfaction outcome & $84.6 \%$ & $93.3 \%$ & 0.841 \\
\hline $\begin{array}{l}\text { Mean (range) difference of haemoglobin levels in pre- and } \\
\text { post-hysteroscopic resection }(\mathrm{g} / \mathrm{L}) \ddagger\end{array}$ & $+21.5(+1$ to +44$)$ & $+17.0(-4$ to +40$)$ & 0.235 \\
\hline Satisfaction outcome when uterine fibroid protrusion $>60 \%$ & $100 \%$ & $100 \%$ & - \\
\hline $\begin{array}{l}\text { Glycine was used } \\
+ \text { Normal saline was used } \\
+n=9 \text { in both groups }\end{array}$ & & & \\
\hline
\end{tabular}


with blood results was $119 \mathrm{~g} / \mathrm{L}$ (range, 91-137 g/L). The difference between pre- and post-haemoglobin level in the conventional resection group was +21.5 $\mathrm{g} / \mathrm{L}$ (range, +1 to $+44 \mathrm{~g} / \mathrm{L}$ ). In the IUM group, two patients were excluded from this part of the analysis as the indication for surgery was post-menopausal bleeding, not menorrhagia. For the remaining 11 patients, the mean preoperative haemoglobin level was $99.1 \mathrm{~g} / \mathrm{L}$ (range, 62-120 g/L). The mean postoperative haemoglobin level among the nine patients in the IUM group who returned with results was $108.8 \mathrm{~g} / \mathrm{L}$ (range, 90-124 g/L). The mean improvement in haemoglobin level was $+17.0 \mathrm{~g} / \mathrm{L}$ (range, -4 to $+40 \mathrm{~g} / \mathrm{L}$ ). There was no significant difference between the change in haemoglobin level pre- and post-operatively between the IUM and conventional groups $(\mathrm{P}=0.235$, Mann-Whitney test; Fig 2).

For both techniques, each group had two patients in whom fibroid protrusion was $<60 \%$ within the uterine cavity, with the remaining patients all having $>60 \%$ protrusion. Of those with $<60 \%$ protrusion, each group had one $(50 \%)$ of two patients who was satisfied with the procedure. In those with $>60 \%, 100 \%$ of patients were satisfied $(n=10$ for conventional group and $n=11$ for IUM group; Table 1).

The data were further divided into groups of small and large fibroids with the cut-off of $3.0 \mathrm{~cm}$ to differentiate the two groups. With regard to small fibroids of $\leq 3.0 \mathrm{~cm}$, the mean duration of procedure was significantly reduced among those using the IUM system (mean, 27.6 mins vs 53.4 mins; $\mathrm{P}=0.019$ ), but fluid deficit was significantly greater (mean, $634.4 \mathrm{~mL}$ using IUM vs $80 \mathrm{~mL}$ using conventional technique; $\mathrm{P}=0.019$; Table 2). There was no statistical difference in overall satisfaction for the two methods. When the procedure involved larger fibroids $(>3.0 \mathrm{~cm})$, there was no difference in operating time $(\mathrm{P}=0.527)$ or patient satisfaction $(\mathrm{P}=0.788)$ between the two methods but considerably more fluid deficit was again generated using the IUM system (mean, 328.6
$\mathrm{mL}$ using conventional technique vs $1839 \mathrm{~mL}$ using IUM; P=0.024; Table 2).

When we reviewed the data for all patients who underwent submucosal fibroid resection using the IUM technique, the procedural time was significantly reduced, while fluid deficit had a lowering trend with smaller fibroids compared with larger fibroids. Regardless of fibroid size, however, there was no significant difference in overall patient satisfaction $(\mathrm{P}=0.710$; Table 3$)$.

\section{Discussion}

Hysteroscopic surgery using hysteroscopic monopolar loop resection has always been the conventional method to resect submucosal fibroids.

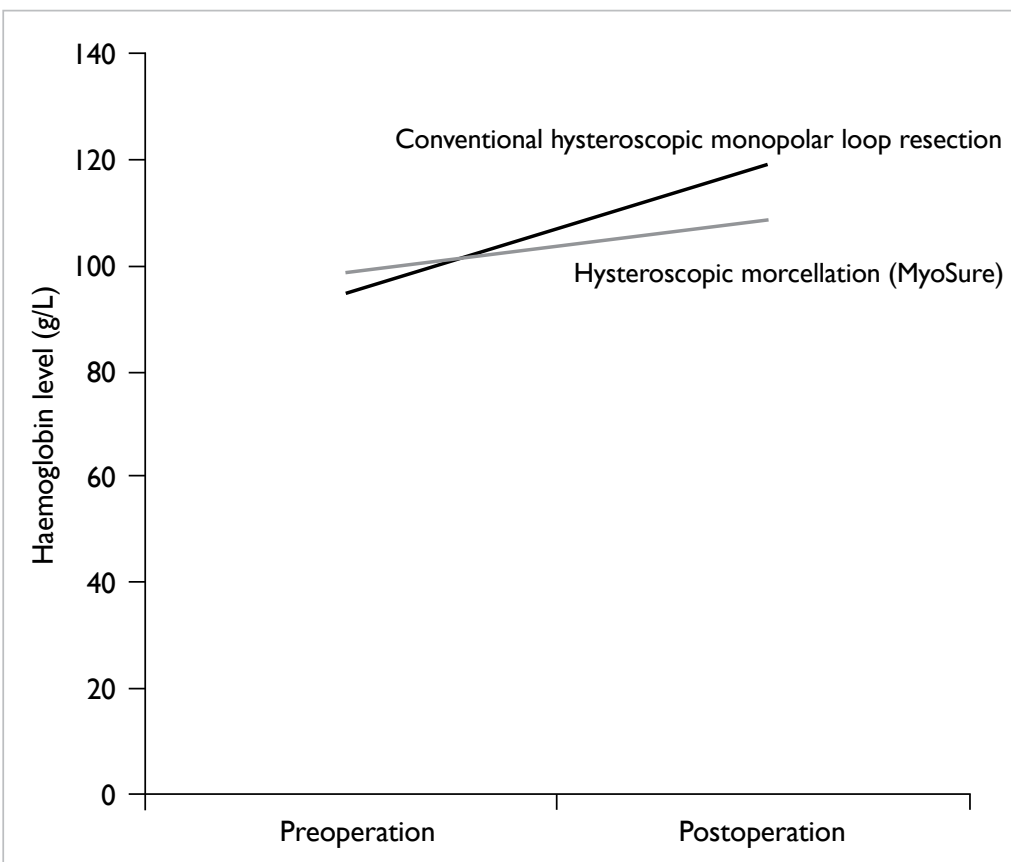

FIG 2. Change in haemoglobin level in pre- and post-hysteroscopic myomectomy

TABLE 2. Comparison of small and large fibroids using the two techniques

\begin{tabular}{lccc}
\hline Fibroid & Conventional technique & MyoSure technique & P value \\
\hline Small $(\leq 3 \mathrm{~cm})$ & $(\mathrm{n}=5)$ & $(\mathrm{n}=9)$ & 0.019 \\
\hline Duration of procedure $($ mins) & $53.4(29-76)$ & $27.6(14-45)$ & 0.019 \\
\hline Fluid deficit $(\mathrm{mL})$ & $80(0-200)$ & $634.4(40-1600)$ & 0.797 \\
\hline Patient satisfaction & $4(80 \%)$ & $9(100 \%)$ & 0.527 \\
\hline Large $(>3 \mathrm{~cm})$ & $(\mathrm{n}=7)$ & $(\mathrm{n}=4)$ & 0.024 \\
Duration of procedure $($ mins) & $65(39-102)$ & $57(40-66)$ & 0.788 \\
\hline Fluid deficit $(\mathrm{mL})$ & $328.6(0-1000)$ & $3(759(556-2600)$ & \\
\hline Patient satisfaction & $6(85.7 \%)$ & & \\
\hline
\end{tabular}


TABLE 3. Comparison of outcome for fibroids of $\leq 3 \mathrm{~cm}$ with fibroids of $>3 \mathrm{~cm}$

\begin{tabular}{lccc}
\hline MyoSure & Fibroids $\leq \mathbf{3 . 0} \mathbf{~ c m ~} \mathbf{( n = 9 )}$ & Fibroids $>\mathbf{3 . 0} \mathbf{~ c m ~} \mathbf{( n = 4 )}$ & P value \\
\hline Duration of procedure (mins) & $27.6(14-45)$ & $57(40-66)$ & 0.006 \\
Fluid deficit $(\mathrm{mL})$ & $634.4(40-1600)$ & $1839(556-2600)$ & 0.076 \\
Patient satisfaction & $9(100 \%)$ & $3(75 \%)$ & 0.710 \\
Complication & 0 & 0 & - \\
\hline
\end{tabular}

Recently, hysteroscopic intrauterine morcellators such as MyoSure have been increasingly used as an alternative. Reports have suggested that such techniques to remove submucosal fibroids and polyps are as effective as the conventional hysteroscopic resection while fibroid symptomrelated quality of life is improved and the recurrence of endometrial polyp reduced., ${ }^{2,3}$ Reports have suggested that IUM may be associated with adverse complications such as bowel damage, hysterectomy, uterine perforation, and pelvic infection, but an adverse event complication rate of $<1 \%$ for hysteroscopic morcellation technique is lower than that for conventional electrocautery. ${ }^{4}$ Other reports have suggested additional benefits such as reduction in instances of uterine perforation, cervical dilation, thermal bowel injury, and intrauterine adhesions when comparing the use of such a device with conventional methods. Operating time, fluid absorption, and the need for a second operation may also be reduced. . $^{5-7}$

Despite its increasing popularity worldwide, the IUM technique remains a relatively new concept within the Chinese population. In this retrospective review, among the 13 patients who underwent IUM for the management of submucosal fibroids, none developed intra-operative complications or postoperative complications that could lead to an extended hospital stay.

Excessive fluid deficit and subsequent fluid absorption remains a concern with hysteroscopic surgery. Electrolyte imbalance as well as cardiac collapse and death can occur in severe cases. This is more likely if hypotonic glycine is used as the uterine distention medium: normal saline reduces such risks. ${ }^{8}$ In our study, the IUM technique was associated with significantly higher fluid deficit regardless of fibroid size being morcellated. One explanation of this is the fast fluid pumping device that is used with the IUM. High fluid flow within the cavity is important to maintain a clear view during the morcellation procedure and to maintain a high intrauterine pressure to prevent bleeding during the myoma morcellation process. Normal saline was used in the IUM technique instead of glycine (which was used in conventional technique). Despite its significantly higher fluid deficit, no patients experienced any associated complications. ${ }^{9}$ In both techniques and regardless of the size of the submucosal fibroids, the total amount of fluid deficit remained within or just above the maximum limit of $2500 \mathrm{~mL}$ of saline or 1000 mL glycine set by AAGL (American Association of Gynecologic Laparoscopists). ${ }^{10}$ One patient who underwent IUM had $100 \mathrm{~mL}(2600 \mathrm{~mL})$ above the recommended maximum fluid deficit limit. This was due to the additional time required to completely resect a large $6-\mathrm{cm}$ fibroid and avoided a second operation. This patient recovered well and did not have any complications. Hence despite the excessive fluid deficit, IUM remains a safe procedure.

When the technicalities of both techniques were compared, the total time required for the operation was significantly reduced when the IUM technique was used. This was particularly significant if the fibroid size was $\leq 3 \mathrm{~cm}$ but not if the fibroid was $>3 \mathrm{~cm} ; 3 \mathrm{~cm}$ was chosen as the cut-off between small and large fibroid as previous studies have already shown morcellation of submucosal fibroids of $\leq 3.0 \mathrm{~cm}$ to be safe and effective. ${ }^{11,12}$ One of the main reasons for the reduced operating time was the constant suction mechanism at the morcellator blade of the IUM device. This suction constantly removes resected material to maintain a clear view of the uterine cavity. The material is collected directly into the Aquilex Fluid Control System that is required for the MyoSure IUM device. As a result, unlike the conventional method, the need to constantly remove fibroid chips during the procedure is avoided and hence operating time is reduced. With the largersized fibroids, the time needed to morcellate the large fibroid will still be considerable so there is a smaller difference compared with conventional methods. One may suggest that the reduced time difference may be due to the experience of the operator, as the IUM is a new technique within our department. Previous study has suggested that both experienced operators and training doctors favour the morcellation technique and the learning curve is minimal. ${ }^{12}$ Familiarisation with the setup of the system, the technique of hysteroscopic morcellation, management of a loose cervix that can cause fluid leakage as well as fluid control to maintain haemostasis versus a clear visual field remain a challenge. Given more experience with the IUM system, reduced operating times will become more significant. This proposed reduction in operating time will benefit both patient (eg 
anaesthetic exposure) and the institution (reduced waiting time for operation). Other potential benefits described by other studies such as reduced uterine perforation, cervical dilation, thermal bowel injury, and intrauterine adhesions $\mathrm{s}^{5-7}$ cannot be determined given the small number of cases performed so far. Nonetheless, there has been one case of uterine and subsequent bowel perforation with the conventional technique and none with the IUM technique in this study.

Patient satisfaction in terms of reduced or improved menstrual symptoms showed no significant difference at 3 months' follow-up when the IUM technique was compared with the conventional technique. Haemoglobin levels improved following hysteroscopic resection of fibroid regardless of the technique used, with no significant difference in improvement between the two groups. Patient satisfaction again showed no statistically significant difference regardless of the size of the submucosal fibroids, suggesting that the IUM technique can be applied to large fibroids. If cases were carefully selected and only submucous fibroids with less than $50 \%$ of the contents intramural were surgically resected as suggested by Di Spiezio Sardo et $\mathrm{al}^{7}$ the satisfaction rate would remain the same between the two techniques, that is both achieved $100 \%$ satisfaction.

\section{Limitations}

Although results regarding safety, effectiveness, and benefits of the IUM technique appear to be promising, this study remains a preliminary overview as the strength of the evidence is limited by the small number of cases. The limited sample may not be representative of the general population and the two groups using different techniques may not be comparable. As a result of the small numbers, this in itself and the non-parametric test used due to the lack of numbers reduce its statistical power. Limitations also arise when the skill of the surgeon varies and the total operating time incorporates the time for preparation and setting up for the procedure. The latter may cause discrepancy in the actual total operating time. Further prospective studies or randomised controlled trials with more subjects, a limited number of surgeons with similar hysteroscopic skills, and a more accurate surgical time measurement would be more beneficial. More standardised outcome measures using a combination of haemoglobin improvement, menstrual chart, and/ or patient satisfaction surveys may also improve the strength of future studies.

\section{Conclusions}

This retrospective review suggests that hysteroscopic IUM is a safe and effective technique for management of menorrhagia secondary to submucosal fibroid.
Preliminary data suggest the technique to be as safe and effective as, and less time-consuming than, conventional techniques. Maximum benefit would be achieved if submucosal fibroid cases for IUM resection were carefully selected, with particular reference to patients in whom $>50 \%$ of the fibroid protrudes into the uterine cavity and where maximum diameter is $\leq 3 \mathrm{~cm}$.

\section{Declaration}

No conflicts of interest were declared by authors.

\section{References}

1. Puri K, Famuyide AO, Erwin PJ, Stewart EA, LaughlinTommaso SK. Submucosal fibroids and the relation to heavy menstrual bleeding and anemia. Am J Obstet Gynecol 2014;210:38.e1-7.

2. Rubino RJ, Lukes AS. Twelve-month outcomes for patients undergoing hysteroscopic morcellation of uterine polyps and myomas in an office or ambulatory surgical center. J Minim Invasive Gynecol 2015;22:285-90.

3. AlHilli MM, Nixon KE, Hopkins MR, Weaver AL, Laughlin-Tommaso SK, Famuyide AO. Long-term outcomes after intrauterine morcellation vs hysteroscopic resection of endometrial polyps. J Minim Invasive Gynecol 2013;20:215-21.

4. Haber K, Hawkins E, Levie M, Chudnoff S. Hysteroscopic morcellation: review of the manufacturer and user facility device experience (MAUDE) database. J Minim Invasive Gynecol 2015;22:110-4.

5. Wamsteker K, Emanuel MH, de Kruif JH. Transcervical hysteroscopic resection of submucous fibroids for abnormal uterine bleeding: results regarding the degree of intramural extension. Obstet Gynecol 1993;8:736-40.

6. Stamatellos I, Apostolides A, Tantis A, Stamatopoulos P, Bontis J. Fertility rates after hysteroscopic treatment of submucous fibroids depending on their type. Gynecol Surg 2006;3:206-10.

7. Di Spiezio Sardo A, Mazzon I, Bramante S, et al. Hysteroscopic myomectomy: a comprehensive review of surgical techniques. Hum Reprod Update 2008;14:101-19.

8. Tarneja P, Tarneja VK, Duggal BS. Complications of hysteroscopy surgery. Medical Journal Armed Forces India 2002;58:331-4.

9. Isaacson $\mathrm{KB}$, Olive $\mathrm{DL}$. Operative hysteroscopy in physiologic distention media. J Am Assoc Gynecol Laparosc 1999;6:113-8.

10. AAGL Advancing Minimally Invasive Gynecology Worldwide, Munro MG, Storz K, Abbott JA, et al. AAGL Practice Report: Practice Guidelines for the Management of Hysteroscopic Distending Media: (Replaces Hysteroscopic Fluid Monitoring Guidelines. J Am Assoc Gynecol Laparosc. 2000;7:167-168.). J Minim Invasive Gynecol 2013;20:137-48.

11. Hamerlynck TWO, Dietz V, Schoot BC. Clinical implementation of the hysteroscopic morcellator for the removal of intrauterine myomas and polyps. A retrospective descriptive study. Gynecol Surg 2011;8:193-6.

12. Emanuel $\mathrm{MH}$, Wamsteker $\mathrm{K}$. The Intra Uterine Morcellator: a new hysteroscopic operating technique to remove intrauterine polyps and myomas. J Minim Invasive Gynecol 2005;12:62-6. 